\title{
CD24+SSEA4+cells in Ovarian Carcinoma Cells Demonstrated the Characteristics as Cancer Stem Cells
}

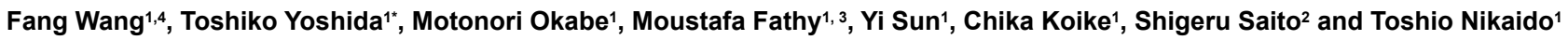

${ }^{1}$ Department of Regenerative Medicine, Graduate School of Medicine and Pharmaceutical Sciences, University of Toyama, Japan

${ }^{2}$ Department of Obstetrics and Gynecology, Graduate School of Medicine and Pharmaceutical Sciences, University of Toyama, Japan

${ }^{3}$ Department of Biochemistry, Faculty of Pharmacy, Minia University, Japan

${ }^{4}$ Department of Anesthesiology, Huashan Hospital of Fundan University, Japan

\section{Abstract}

Object: Cancer stem cells (CSCs) have recently started attracting attention as targets cancer treatment Despite many studies, markers of effective CSCs of heterogeneous ovarian carcinoma (OC) that can be directly used for medical treatment have not yet been reported. The aim of this research was to identify a new combination of surface markers for human ovarian CSCs.

Methods: Primary serous adenocarcinomas were obtained from patients with OC. FACS were used to decide cell surface marker to characterize and isolate cancer stem cells from primary human cancer tissues. RT-PCR methods were used for estimating the expression levels of mRNA, and immunohistochemistry confirmed the produce of the stemness relative protein after cell sorting. Xenograft model was used for the ability of tumorogenesis.

Results: We found that the cultured cells strongly expressed stemness genes such as c-myc, oct3/4, sox2, nanog, abcg-2, and bmi-1. They also expressed surface markers such as CD24, SSEA4, CD133, and CD47. The sub-population of CD24+ SSEA4+ double positive cells showed strong expression of Oct-4, and was able to form spheres much more than CD24-SSEA4+ cells, CD24+SSEA4-cells or CD24- SSEA4-double negative cells. Only 5 CD24+SSEA4+ cells formed spheres within 3 weeks. This sub-population formed the highest number of colonies in the soft agar colony assay. The CD24+SSEA4+ cells showed better growth in the presence of cisplatin than the other sub-populations. CD24+SSEA4+ cells were tumorigenic in all scid mice (4/4) within 2 months, with an injection of 100 cells/mouse.

Conclusion: These results suggested that CD24+SSEA4+double positive cells have characteristics similar to that of human ovarian tumor CSCs. It might be possible to develop effective new clinical therapies for human ovarian cancer $(\mathrm{OC})$ using these cells as targets.

Keywords: Cancer stem cells (CSCs); Ovarian carcinoma; Cell surface marker; CD24; SSEA4; Human; Xenograft model

\section{Introduction}

In solid tumors, not all the cancer cells have a similar ability to drive tumor formation, and functional heterogeneity in the tumor has been hypothesized as to account for this [1]. This has led to the hypothesis concerning cancer stem cells (CSCs). CSCs are a minority cell type in tumors, which retains the capacity for cell renewal as well as for differentiation into multiple cell types through asymmetric division. Thus, CSCs can regenerate the entire tumor phenotype and subsequently lead to metastasis. Although many methods have been proposed for identifying and isolating CSCs, none are available for isolating CSCs from solid tumors [2].

Successful cancer treatments shrink the bulk of tumor cells, but often fail to eliminate the CSCs [3,4]. This leads to recurrence of the cancer. Thus, eradication of these CSCs can lead to successful treatment of the patient. In order to target CSCs, it is important to better characterize them by defining specific markers to identify them [5]. However, isolating CSCs is difficult because of the lack of specific cell surface markers in solid tumors [6]. Initial in vitro studies in solid tumor CSC biology have focused on the isolation and propagation of cells with CSC-like properties from breast tumors. A sub-population of CD44+CD24-cells was isolated [7] through the expression of cell surface markers [8].

In gynecologic neoplasms, several studies have indicated that CSCs also promote ovarian tumor growth and metastasis. [9] had shown that single clones which established in animal models are similar to those in the human disease isolated from the ascites of a patient with OC showed stem-like properties. A sub-population of cells that was identified was shown to be tumorigenic in vivo [10]. Similarly, [11] identified tumorigenic populations of cells from human OC cell lines, based on CD133 expression. In a study using primary human ovarian tumor tissues, tumor cells were propagated as anchorageindependent spheroids in specialized media conditions originally designed for maintaining neuronal stem cells [12]. Using this method, the CD44+CD117+ cells were identified as an OC-initiating population capable of creating histologically similar tumors in vivo. In a different study, CD44+/MyD88+ cells were found to be an ovarian tumor cell population with some stem cell-like properties [13]. Additionally, Ince et al. [14] used a differential culture method to isolate two separate

*Corresponding author: Toshiko Yoshida, Department of Regenerative Medicine, Graduate School of Medicine and Pharmaceutical Sciences University of Toyama, 2630 Sugitani, Toyama 9300194, Japan, Tel: 817647211 Fax: 81764345011; E-mail: yosidato@med.u-toyama.ac.jp

Received February 14, 2017; Accepted March 15, 2017; Published March 20 2017

Citation: Wang F, Yoshida T, Okabe M, Fathy M, Sun Y, et al. (2017) CD24+SSEA4+cells in Ovarian Carcinoma Cells Demonstrated the Characteristics as Cancer Stem Cells. J Cancer Sci Ther 9: 343-352. doi: 10.4172/1948-5956.1000440

Copyright: () 2017 Wang F, et al. This is an open-access article distributed under the terms of the Creative Commons Attribution License, which permits unrestricted use, distribution, and reproduction in any medium, provided the original author and source are credited. 
Citation: Wang F, Yoshida T, Okabe M, Fathy M, Sun Y, et al. (2017) CD24+SSEA4+cells in Ovarian Carcinoma Cells Demonstrated the Characteristics as Cancer Stem Cells. J Cancer Sci Ther 9: 343-352. doi: 10.4172/1948-5956.1000440

populations from transformed mammalian epithelial cells that had been derived from a single source. These phenomena suggested that ovarian tumors comprised heterogeneous cells [15].

As mentioned previously, research on CSCs in OC have advanced rapidly in recent years. These studies emphasized the important issue of heterogeneous OCs. Markers of effective CSCs directly associated with medical treatment have not yet been reported.

In this study, we isolated cancer cells from primary human OC specimens that were xenotransplantated, and defined their heterogenic characteristics after selection using cell surface markers. The aim of this experiment was to identify CSCs in OCs as the first step towards developing a new treatment.

\section{Materials and Methods}

\section{Tissues from human ovarian tumors}

Tumor tissue specimens, categorized as serous adenocarcinomas, were obtained from different patients with OC $(n=4)$ during surgical procedures. The study was approved by the Research Ethics Committee of the University of Toyama (Approval number: 20-21) and informed consent was obtained from all the patients.

\section{Isolation of cells}

After the tissue pieces were digested with $0.4 \%$ collagenase for $30 \mathrm{~min}$ at $37^{\circ} \mathrm{C}$, filtered through a gauze filter, they were centrifuged at 2,500 rpm for $5 \mathrm{~min}$, and washed twice with Dulbecco's modified Eagle's medium (DMEM, Sigma-Aldrich Co., St Louis, MO, USA). The cell pellet was collected and suspended in DMEM-F12 supplemented with $20 \%$ fetal bovine serum (FBS, Biosolutions International, Melbourne, Australia), 1\% L-glutamine (Sigma-Aldrich Co.), and 1\% penicillin-streptomycin mixture (Invitrogen, Grand Island, NY, USA). The suspended cells were seeded in $10 \mathrm{~cm}^{2}$ dishes at $37^{\circ} \mathrm{C}$ in a $5 \% \mathrm{CO}_{2}$ humidified incubator. After 1 or 2 days, the floating cells were discarded, the attached cells were retained, and the culture was continued.

\section{Cell proliferation assay}

The cells were seeded at a density of 1000 cells/well into 6-well plates. The medium was changed every $24 \mathrm{~h}$, and the cells were counted on alternate days for 12 days. The cell proliferation, the sphere formation, and the colony assays were all performed in triplicate.

\section{Flow cytometric analysis}

After the cells were blocked with 5\% bovine serum albumin (BSA) in phosphate-buffered saline (PBS), they were stained with CD24 (anti-CD24-FE,), SSEA4 (anti-SSEA4-FITC), SSEA1 (anti-SSEA1-PE), CD133 (anti-CD133-PE), CD47 (anti-CD47-PE), TRA-1-60 (antiTRA-1-60-FITC), TRA-1-81 (anti-TRA-1-81-FITC), or Oct3/4 (antiOct3/4-PE). Flow cytometry was performed using a FACS Canto II.

\section{Reverse transcription-polymerase chain reaction (RT-PCR)}

Total RNA was extracted from the cells using Isogen (Nippon Gene, Tokyo, Japan), according to the manufacturer's instructions. Aliquots of $1 \mu \mathrm{g}$ of total RNA were treated with $0.1 \mathrm{U} / \mu \mathrm{l}$ deoxy ribonuclease I (DNase I, Sigma-Aldrich Co.) at $37^{\circ} \mathrm{C}$ for $15 \mathrm{~min}$. cDNAs were synthesized using $0.5 \mu \mathrm{g}$ DNase I-treated RNA using a Rever Tra Ace qPCR RT Kit (Toyobo Co., Ltd., Osaka, Japan). The resulting cDNAs were then subjected to PCR using a Taq DNA polymerase kit (Qiagen, Hilden, Germany) using the primers (Invitrogen) for $c-m y c$, sox-2, nanog, oct3/4, abcg-2, bmi-1, ck-18, nestin, klf-4, and B-actin (Table 1).

\begin{tabular}{|c|c|c|c|}
\hline Gene name & F/R & Sequence (5'-3') & Size (bp) \\
\hline \multirow[t]{2}{*}{$c-m y c$} & $\mathrm{~F}$ & GATTCTCTGTCTCCTCGACGGAG & \multirow[t]{2}{*}{273} \\
\hline & $\mathrm{R}$ & GCGCTGCGTAGTTGTGCTGATGTG & \\
\hline \multirow[t]{2}{*}{ sox-2 } & $\mathrm{F}$ & AGTCTCCAAGCGACGAAAAA & \multirow[t]{2}{*}{410} \\
\hline & $\mathrm{R}$ & GGAAAGTTGGGATCGAACAA & \\
\hline \multirow[t]{2}{*}{ nanog } & $\mathrm{F}$ & CAGAAGGCCTCAGCACCTAC & \multirow[t]{2}{*}{216} \\
\hline & $\mathrm{R}$ & CTGTTCCAGGCCTGATTGTT & \\
\hline \multirow[t]{2}{*}{ oct-4 } & $\mathrm{F}$ & GAAGCTGGAGAAGGAGAAGCTG & \multirow[t]{2}{*}{224} \\
\hline & $\mathrm{R}$ & CAAGGGCCGCAGCTTACACATGTTC & \\
\hline \multirow[t]{2}{*}{$a b c g-2$} & $\mathrm{~F}$ & TCAGGTAGGCAATTGTGAGG & \multirow[t]{2}{*}{210} \\
\hline & $\mathrm{R}$ & CTTCAGCATTCCACGATATGG & \\
\hline \multirow[t]{2}{*}{$b m i--1$} & $\mathrm{~F}$ & AATCTAAGGAGGAGGTGA & \multirow[t]{2}{*}{370} \\
\hline & $\mathrm{R}$ & CAAACAAGAAGAGGTGGA & \\
\hline \multirow[t]{2}{*}{$c k-18$} & $\mathrm{~F}$ & TGGTCACCACACAGTCTGCT & \multirow[t]{2}{*}{357} \\
\hline & $\mathrm{R}$ & CCAAGGCATCACCAAGATTA & \\
\hline \multirow[t]{2}{*}{ nestin } & $\mathrm{F}$ & AGAGGGGAATTCCTGGAG & \multirow[t]{2}{*}{410} \\
\hline & $\mathrm{R}$ & CTGAGGACCAGGACTCTCTA & \\
\hline \multirow[t]{2}{*}{$k l f-4$} & $\mathrm{~F}$ & GTTTTGAGGAAGTGCTGAG & \multirow[t]{2}{*}{332} \\
\hline & $\mathrm{R}$ & CAGTCACAGTGGTAAGGTTT & \\
\hline \multirow[t]{2}{*}{$\beta$-actin } & $\mathrm{F}$ & CGGGACCTGACTGACTAC & \multirow[t]{2}{*}{252} \\
\hline & $\mathrm{R}$ & GAAGGAAAGGCTGGAAGAG & \\
\hline
\end{tabular}

Table 1: RT-PCR primer sequences. Transfection factor relative with stemness gene, and stemness gene of neuron.

\section{Immunocytochemistry}

Cytospin samples (Cytospin 4, Thermo Scientific, USA) from OC cells were stained with primary antibodies against CD24 (Abcam), SSEA-4, SSEA-1, c-Myc, Sox2, Nanog, Oct3/4, ABCG-2, Klf-4, and CK18 (Santa Cruz Biotechnology) and examined by microscope (Leika DMRBE) under fluorescence.

\section{Chemotherapy resistance assay}

The cells were either untreated or treated with cisplatin (Kyowa Hakko Kogyo, Japan) at concentrations of 30, 40, 75, or $100 \mu \mathrm{M}$. After $24 \mathrm{~h}$, the cell viability was analyzed using the Cell Counting Kit- 8 (CCK-8, Dojindo, Kumamoto, Japan). The percentage of viable cells was calculated using the following formula: viability $(\%)=(\mathrm{X} \times 10 \%) / \mathrm{Y}$; Where $\mathrm{X}$ is the absorbance of the treated cells and $\mathrm{Y}$ is that of the salinecontaining medium-treated group.

\section{In vivo serial transplantation of human OC cells}

Male and female nude mice weighing $22 \mathrm{~g} \pm 2 \mathrm{~g}$ were provided by Japan SLC, Inc. Animal care and experimental protocols were performed in accordance with the guidelines established by the Experimental Animal Center, University of Toyama (A2012 MED-61).

\section{Cell sorting with magnetic-activated cell sorting (MACS) separator}

OCs were isolated using MACS (Magnetic Cell Sorting, Miltenyi Biotec.) with CD24+ and/ or SSEA4+ cells. The cells were blocked with the FcR Blocking Reagent (Phosphate buffer saline; PBS pH 7.2 and $0.5 \% \mathrm{BSA}$ ) and $2 \mathrm{mM}$ EDTA and then diluted to $1: 20$ with the auto 
MACS Rinsing Solution (Miltenyi Biotech, \#130-091-222) containing the MACS BSA Stock solution (Miltenyi Biotec, \#130-091-376).

\section{Sphere formation}

Cells were seeded at a density of $1 \times 10^{3}$ cells/well into 24 -well ultralow attachment dishes (Corning, NY, USA) in serum-free media, and cultured at $37^{\circ} \mathrm{C}$ in $5 \% \mathrm{CO}_{2}$ in a humidified incubator for 4 days. The number of spheres formed was counted using a microscope (ECLIPSE TE2000-U; Nikon).

\section{Anchorage independent growth assay}

Cells were seeded at a density of $4 \times 10^{4}$ cells/dish into $60-\mathrm{mm}$ dishes in a suspension of $0.4 \%$ Noble agar (Difco, Becton, Dickinson and Company, MD, USA) with a supplemented medium on top of a bed of $0.5 \%$ Bacto Agar (Difco, Becton, Dickinson and company) in the same medium. The number of colonies larger than $500 \mu \mathrm{m}$ that had formed after two weeks of incubation was counted.

\section{Pathology and immunohistochemistry of the formed tumors}

The formed tumors were stained with hematoxylin-eosin (Sakura Fine Tech., Tokyo, Japan) or analyzed with immunohistochemistry using stains against CD24, CD44, and SSEA4.

\section{Xenograft model in nude and scid mice}

In each group, the cells were diluted to different concentrations (5 $\times 10^{5}, 5 \times 10^{4}, 5 \times 10^{3}, 5 \times 10^{2}$, and $1 \times 10^{2}$ cells $/ \mathrm{ml}$ ) in a BD Matrigel medium (BD Bioscience, MA, USA). Cells $(0.2 \mathrm{ml})$ were injected subcutaneously into the left (CD24+SSEA4+) and right (CD24-SSEA4+ or CD24-SSEA4-) flanks of nude or scid mice (Japan SLC, Charles River, Yokohama, Japan), respectively. The injected mice were checked for tumor formation for up to 10 weeks.

\section{Statistical analysis}

Data were presented as the mean \pm standard error (SE). Bar graphs were generated and statistical analyses were performed using the Statistics software (SPSS Statistics ver. 20 for Mac; IBM, Tokyo, Japan). Differences between groups were considered significant when the probability values $(\mathrm{P})$ were less than 0.05 .

\section{Results}

\section{OC cells expressed several surface markers}

Cells were isolated from primary and serially transplanted tumors. In the primary cells, the rate of expression of CD24, SSEA4, and CD133 was high, while that of the other markers was low (Figure a1), according to the results of the flow cytometry. However, the rates of expression of CD47 and Tra-1-60 in different patients were different. In the serially transplanted cancer cells, the rates of expression of CD24, SSEA4, and CD133 were $14.5 \%, 33 \%$, and $21.1 \%$, respectively (Figure a1 and a2). Although the rate of expression of CD47 was highest in surface markers in the serially transplanted cells, it was very different from that in the primary cells. In contrast, the rates of expression of CD24, SSEA4, and CD133 were not very different from those in the primary cells. The expression rates of SSEA1, Tra-1-60, and Tra-1-81 were below $1.0 \%$. The double positive cells of CD24 and CD133 or CD24 and SSEA4 constituted $5.3 \%$ and $5.6 \%$ of the studied cells, respectively (Figure $1 \mathrm{~b}$ ).

We investigated the expression levels of several stemness genes in OCs, including $c-m y c$, sox-2, nanog, abcg-2, bmi-1, oct 4, nestin, klf4 , and $c k-18$ (Figure 1c). The OCs showed strong expression of many stemness genes. After separating the cells based on the cell surface markers, CD24+ or SSEA4+ cells were found to express the stemness genes stronger than CD24- or SSEA4-cells. CD24- and SSEA4-cells did not express any of the genes, except nestin (Figure 1d). However, the difference in expression between $\mathrm{CD} 133+$ and CD133-cells was not large (Figure 1d).

\section{Proliferation of CD24+SSEA4+ cells after sorting was faster than that of other sub-classes}

Although no differences were observed in the morphology of the different sub-populations, the proliferation rate of CD24+SSEA4+ cells were significantly higher than that of the CD24+SSEA4-, CD24-, or parent cells (Figure $2 \mathrm{a}$ and $2 \mathrm{~b}$ ).

\section{CD24+ SSEA-4+ cells strongly expressed stemness genes}

The CD24+SSEA4+ cells expressed stem cell-related genes more than the unsorted cells (parent cells), while the CD24+SSEA4cells expressed them as much as or a little lesser than the parent cells. In particular, oct 4, sox-2, c-myc, klf-4, abcg-2, and bmi-1 were more highly expressed in CD24+SSEA4+ cells than in CD24+SSEA4-cells (Figure 2c).

The protein expression levels of stemness markers, Oct 3/4, Nanog, Sox-2, Klf-4, c-Myc, and ABCG-2 were assessed in the CD24+SSEA4+ and CD24+SSEA4-cells using immunohistochemical staining. The differentiation markers, ck-18 and vimentin, were found to be expressed in all cells. Stemness markers were also expressed in CD24+SSEA4cells, but with weak signals. The CD24-cells showed no expression of any of the protein markers of stemness (Figure 3).

\section{CD24+SSEA4+ cells reconstituted the heterogeneity of parent cells}

In order to identify the sub-populations that could reconstitute the heterogeneity of the parental cells, the sorted cells were analyzed by flow cytometry using SSEA4 and CD24 antibodies after 2-3 passages. We found that while the CD24+SSEA4+ cells were consistent with the heterogeneous cells in the pre-sorting stage, the other cells were not (Figure 4). Before sorting, CD24+SSEA4+ cells comprised 5.6\%, CD24+SSEA4-cells comprised 14.9\%, CD24-SSEA4+ cells comprised 6.5\%, and CD24-SSEA4-cells comprised $73.0 \%$. After sorting, the cultured cells were analyzed again. Only the cells cultured from CD24+SSEA4+ cells could be separated into four parts similar to the unsorted ones (Figure 4).

\section{CD24+SSEA4+ cells had high potential to form spheres}

The CD24+SSEA4+ cells formed $44 \pm 2$ spheres for $1 \times 10^{3}$ cells, and the average of their diameter was $200 \mu \mathrm{m}$. The CD24+SSEA4cells and the CD24-SSEA4+cells formed $17 \pm 1.5$ and $16 \pm 2$ spheres for $1 \times 10^{3}$ cells, respectively, and the average of their diameter was $120 \mu \mathrm{m}$. CD24-SSEA4-cells formed small spheres under the same experimental conditions. The average diameter of the spheres was 2 $\mu \mathrm{m}$. CD24+SSEA4+cells showed a higher potential to form spheres than the other sub-populations (Figure 5-a1 and a2). The formed spheres were grouped into three types: large, middle, and small ( $\mathrm{L}>100$ $\mu \mathrm{m}>\mathrm{M}>50 \mu \mathrm{m}>\mathrm{S}>20 \mu \mathrm{m})$. The CD24+SSEA4+cells formed the most number of cells, regardless of the size (Figure 5-a3). Surprisingly, only 5 CD24+SSEA4+ cells formed spheres within three weeks (Figure 5-a4).

\section{CD24+SSEA4+cells had high potential to form colonies in soft agar}

The CD24+SSEA4+ and CD24+SSEA4-cells formed $5117 \pm 87$ 
Citation: Wang F, Yoshida T, Okabe M, Fathy M, Sun Y, et al. (2017) CD24+SSEA4+cells in Ovarian Carcinoma Cells Demonstrated the Characteristics as Cancer Stem Cells. J Cancer Sci Ther 9: 343-352. doi: 10.4172/1948-5956.1000440

(a) -1

\begin{tabular}{|c|c|c|c|c|c|c|c|c|}
\hline $\begin{array}{c}\text { Primary } \\
8\end{array}$ & CD24 & SSEA-4 & SSEA-1 & CD133 & CD47 & $\begin{array}{c}\text { TRA-1 } \\
60\end{array}$ & $\begin{array}{c}\text { IRA-1 } \\
81\end{array}$ & OCt3/4 \\
\hline 1 & 18.5 & 52.2 & 3.4 & 29.3 & 1.8 & 0.3 & 0.2 & 7.5 \\
\hline 2 & 11.6 & 32.0 & 0.4 & 27.0 & 29.3 & 40.0 & 0.2 & 4.7 \\
\hline 3 & 20.4 & 19.9 & 1.3 & 28.0 & 25 & 1.9 & 0.1 & 3.0 \\
\hline
\end{tabular}

(b)

(a) -2

\begin{tabular}{|c|c|c|c|c|c|c|c|c|}
\hline $\begin{array}{c}\text { Xeno- } \\
\text { translated } \\
8\end{array}$ & CD24 & SSEA4 & SSEA1 & CD133 & CD47 & $\begin{array}{c}\text { TRA-1 } \\
60\end{array}$ & $\begin{array}{c}\text { TRA-1 } \\
81\end{array}$ & OCt3/4 \\
\hline 1 & 15.3 & 29.3 & 0.5 & 27.4 & 85.2 & 0.6 & 0.6 & 0.8 \\
\hline 2 & 11.3 & 55.3 & 1.0 & 17.2 & 87.6 & 0.8 & 0.6 & 1.0 \\
\hline 3 & 15.9 & 30.5 & 0.2 & 16.5 & 49.5 & 0.1 & 0.1 & 0.1 \\
\hline 4 & 17.6 & 24.40 & 1.6 & 27 & 63.90 & 0.30 & 1.1 & 2 \\
\hline 5 & 12.2 & 25.5 & 0.6 & 17.5 & 55.9 & 0.7 & 10 & 1.4 \\
\hline Average & $\begin{array}{c}14.5 \\
\pm 2.6\end{array}$ & $\begin{array}{c}33 \\
\pm 12.7\end{array}$ & $\begin{array}{c}0.78 \pm \\
0.5 \%\end{array}$ & $\begin{array}{c}21.1 \\
\pm 5.6\end{array}$ & $\begin{array}{c}68.4 \\
\pm 17.2\end{array}$ & $\begin{array}{c}0.5 \\
\pm 29\end{array}$ & $\begin{array}{c}0.7 \\
\pm 4\end{array}$ & $\begin{array}{c}1.1 \\
\pm 7.1\end{array}$ \\
\hline
\end{tabular}

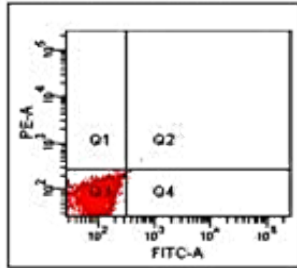

Isotype IgG2C

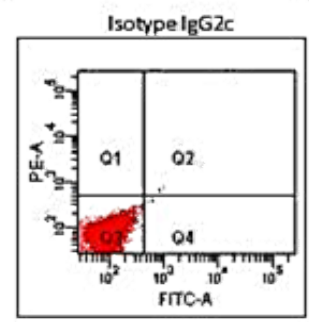

Double staining

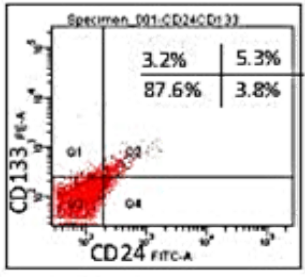

Double staining
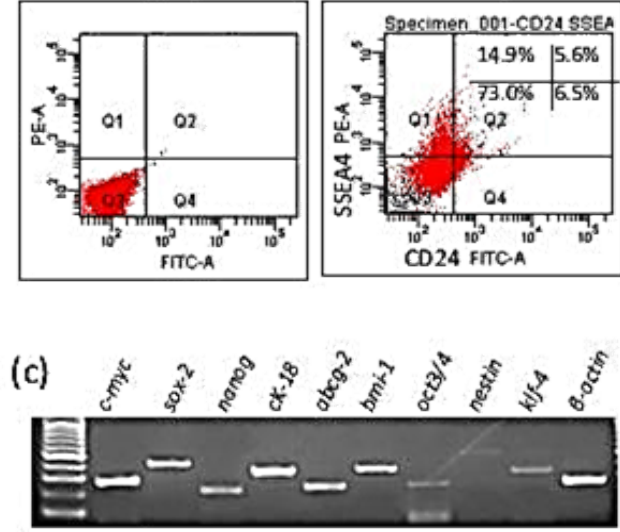

(d)
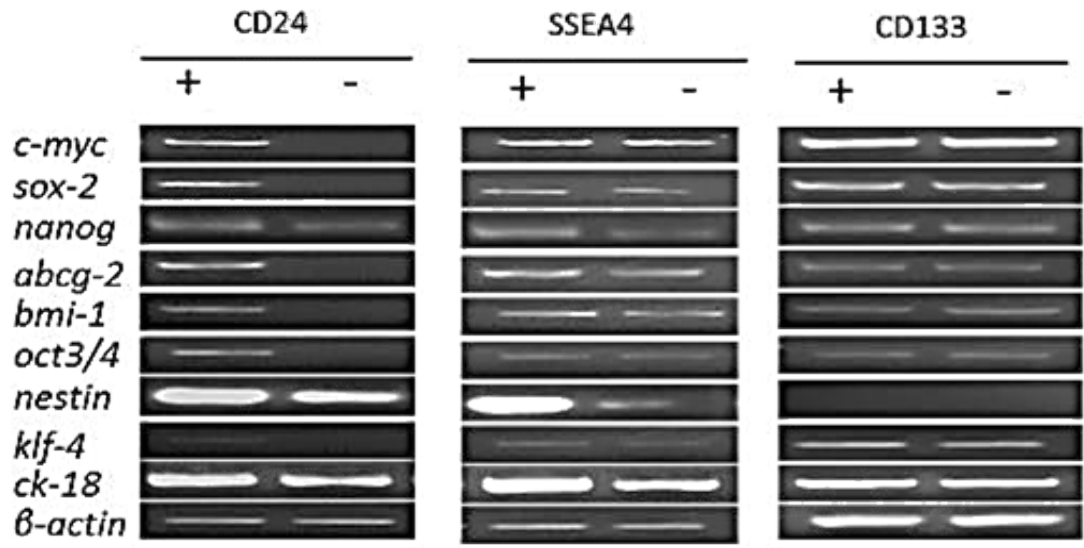

目 ${ }_{\mathrm{CD} 24+} \mathbf{\theta}_{\mathrm{CD} 24-}$

ESSEA4+ QSSEA4.

$\nabla_{133+} \nabla_{133-}$
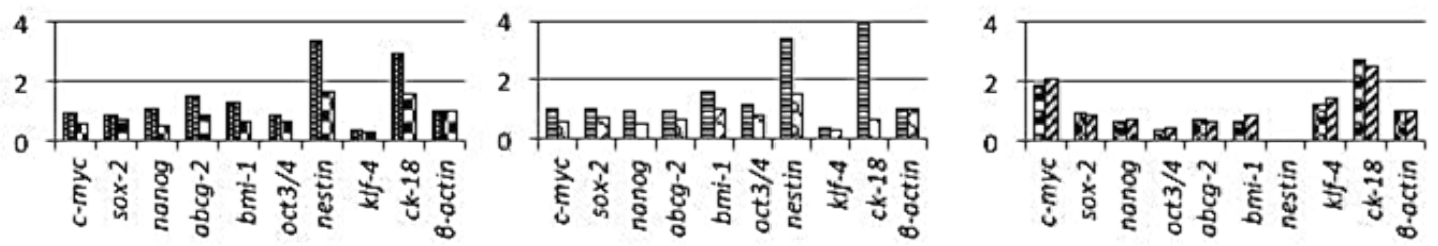

Figure 1: Characteristics of isolated ovarian cancer $(O C)$ cells.

(a)-1: The expression of cell surface marker in the isolated cells from primary ovarian cancer. The expression of CD24, SSEA4, CD133 was high.

(a)-2: The expressions of cell surface marker in isolated cells from serial xenografted ovarian carcinoma cells were analyzed by FACS. The expression of CD24, SSEA4, CD133, CD47 were high, SSEA1, TRA-1-60 and TRA-1-81 were low.

(b) The ratios of sub-populations were measured for CD24 and CD133 or CD24 and SSEA4. It was CD24+SSEA4+: 5.6\%, CD24+SSEA4-:14.9\%, CD24SSEA4-:73.0\%. (c) Conventional RT-PCR analyses of stemness related genes. Many stemness genes (oct 4, c-myc, sox2, klf-4, nanog, abcg-2, bmi-1) were expressed in isolated cells from primary OC cells.

(d) Expression of embryonic and adult stemness related genes by RT-PCR. Relative expression level was formulated as stemness genes/ $\beta$-actin. The compared expression of stemness genes in CD24+ and CD24- cells, SSEA4+ and SSEA4-, or CD133+ and CD133- derived from isolated OC cells. All stemness genes expressed in the CD24+ cells. In contrast, CD24- cells did not express them except $c k-18$ or nestin. The expression of nanog, Oct 4, nestin or klf-4 was stronger on the SSEA4+ cells than SSEA4-cells. There was no big difference between CD133+ and CD133-cells. 


\section{(c) Expression of stemness gene}

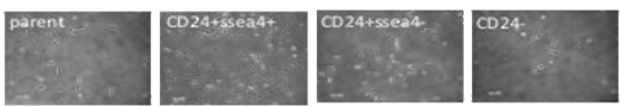

(b)Compare the growth curve with sub population

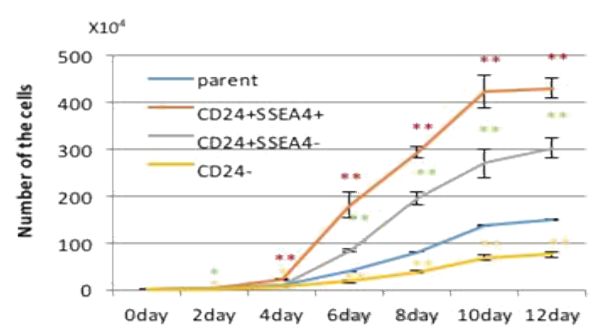

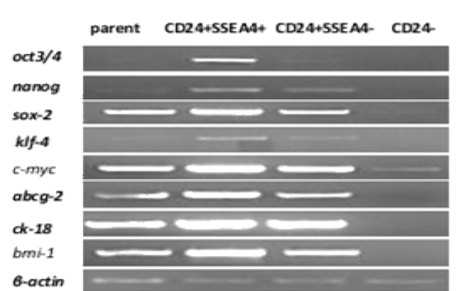

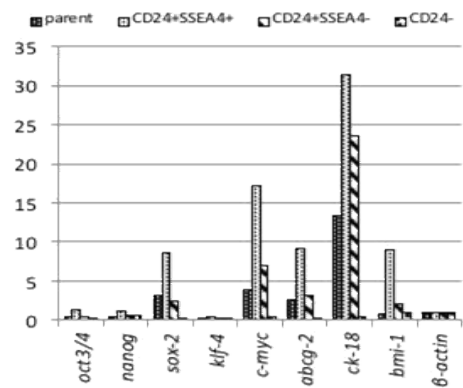

Figure 2: Characteristic differentiation between sub-population cells was compared for CD24+SSEA4+, CD24+SSEA4- and CD24- cells.

(a) Morphology of sub-populations. It was so different the morphology each sub-population, but the concentration was most in the CD24+SSEA4+ cells.

(b) Growth curve. Proliferation of cells of CD24+SSEA4+, CD24+SSEA4- and CD24- sub-populations was compared within 14 days after sorting. The proliferation of CD24+SSEA4+ cells was faster than the other sub-populations $\left({ }^{* *} p<0.01\right)$.

(c) In CD24+SSEA4+ cells expressed all stemness genes strongly. There did not expressed oct 4, nanog or klf-4 in CD24+SSEA4- and parent cells. CD24- cells did not express almost stemness genes except c-myc.

and $3048 \pm 83$ colonies that were larger than $500 \mu \mathrm{m}$ in diameter, respectively. The ability of CD24+SSEA4+ cells to form colonies was 10 times that of the CD24-cells (Figure 5b).

\section{CD24+SSEA4+cells were resistant to cisplatin}

The cells were treated with cisplatin (Kyowa Hakko Kogyo, Japan) at concentrations of $30,40,75$, or $100 \mu \mathrm{M}$. The viability of CD24+SSEA4+cells did not change, despite the concentration of cisplatin used. In contrast, the cells from other sub-populations showed decreased viability as with increasing concentration of cisplatin. No cells were found to survive among the parent cells or CD24-SSEA4-cells at concentrations of cisplatin above $75 \mu \mathrm{M}$ (Figure 5c).

\section{Histopathology of xenografted tumors}

Both the primary patient tumors and xenografted tumors (Figure 6a) were diagnosed as serous adenocarcionomas and exhibited similar pathological characteristics (Hematoxylin and eosin-stained section); however, some of the xenografted tumors appeared more severe than the primary ones. The expression levels of CD24 and SSEA4 in the xenografted tumors (passage 9) were assessed, and it was found that almost all the tumor cells showed strong positive staining.

\section{CD24+SSEA4+cells were highly tumorigenic}

The sorted cells were injected subcutaneously into nude mice. All the mice that received $5 \times 10^{5} \mathrm{CD} 24+\mathrm{SSEA} 4+$ cells formed tumors $(3 / 3)$ at 4 weeks post-injection, while no tumorigenesis was observed in mice injected with $5 \times 10^{5}$ unsorted cells. Mice that received $5 \times 10^{4}$ CD24+SSEA4+cells formed tumors (3/3), while those that received $5 \times 10^{4} \mathrm{CD} 24+$ SSEA4-cells did not. When $1 \times 10^{2}$ cells were injected into the mice, only the mice injected with CD24+SSEA4+cells formed tumors (3/3) at 2 months ( 8 weeks) post-injection. Double negative cells showed no tumors until 10 weeks post-injection (Figure 6-b1). When injected with $1 \times 10^{2}$ cells, the CD24+SSEA4+cells were tumorigenic in all nude mice, while the CD24-cells did not form tumors. The tumors which formed from 100 cells of CD24+SSEA4+ included CD24+ cells and CD24-cells (Figure 6-b2). This observation suggested that CD24+SSEA4+ cells could differentiate into other cell types.

\section{Discussion}

In this study, we described the isolation and characterization of a highly tumorigenic sub-population of cells, CSCs, from human OCs. Five separate criteria have been established for CSCs: a) self-renewal, b) small minority of the total tumor population, c) reproducible tumor phenotype, d) multipotent differentiation into non-tumorigenic cells, and e) distinct cell-surface antigenic phenotype, permitting consistent isolation [16,17]. Two recent commentaries of previously identified CSCs, however, questioned their abilities of self-renewal and multipotency $[18,19]$; in addition, another report suggested possible misinterpretations resulting from engraftment of hematologic CSCs in non-native growth environments [20].

In the present study, we found that the isolated cells expressed stemness genes such as oct 4, sox2, klf, nanog, abcg-2,c-myc, bmi-1, and nestin (Figure 1c). These cells also expressed surface markers associated with stemness, including CD24, SSEA4, CD133, and CD47 (Figure 1a). CD24 is used as a cell surface marker of CSCs in hepatocarcinomas [21], prostate cancers [22], clear cell renal carcinomas [23], and OCs [24]. CD133 is also considered a cell surface marker of CSCs in melanomas, OCs, and other cancers [25-28]. The expression of SSEA-4 was found to increase from normal epithelium to benign cystadenoma to borderline cystadenoma and adenocarcinoma, in both serous and mucinous groups. The loss of or reduction in the expression of SSEA4 was significantly correlated with advanced tumor stage and reduced tumor cell differentiation [29]. Furthermore, it was recently reported that CD44+SSEA4+ was a useful marker for isolating CSCs from oral 
Citation: Wang F, Yoshida T, Okabe M, Fathy M, Sun Y, et al. (2017) CD24+SSEA4+cells in Ovarian Carcinoma Cells Demonstrated the Characteristics as Cancer Stem Cells. J Cancer Sci Ther 9: 343-352. doi: 10.4172/1948-5956.1000440
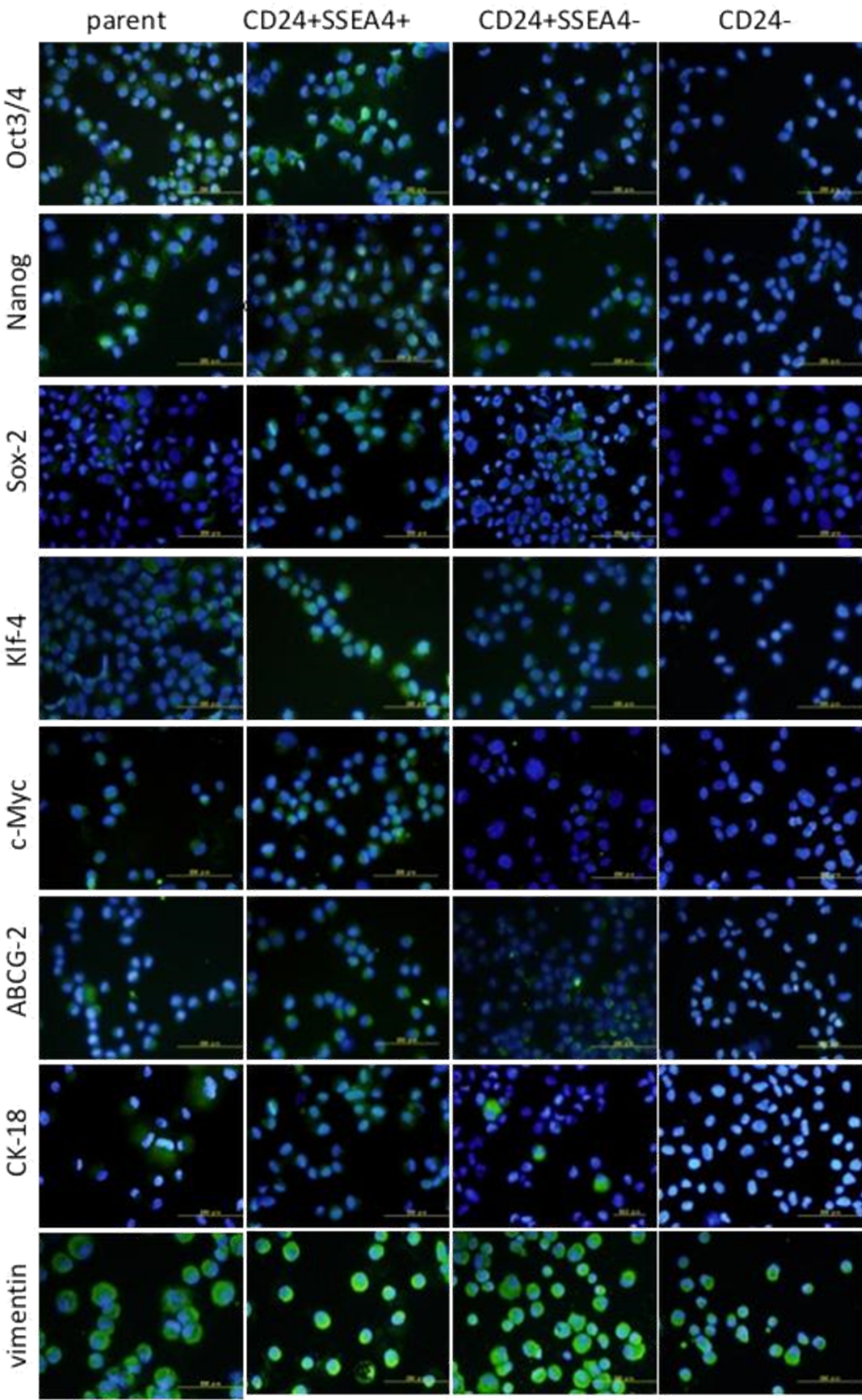

Figure 3: The immunocytochemistry of parent cells, CD24+SSEA4+ cells, CD24+SSEA4- cells and CD24- cells. Oct3/4, Nanog, Sox-2, KIf-4, c-Myc and ABCG-2 were observed in them. The differentiation markers, CK-18 and Vimentin, were seen in all cells. Stemness markers were also expressed in CD24+SSEA4-cells, but with weak signals. CD24-cells showed no expression of the protein markers of stemness.

squamous cell carcinomas [30]. However, no good combination of markers has yet been reported for identifying CSCs that are capable of initiating and metastasizing tumors [31]. We attempted to identify an optimal combination of cell surface markers. First, we measured the rates of expression of stem cell-related genes for each subpopulation of cancer cells using FACS after sorting the cells based on various combinations of cell surface markers. CD133 was reported to be a CSC of primary human OC [32]. Indeed, we found the ratio of 


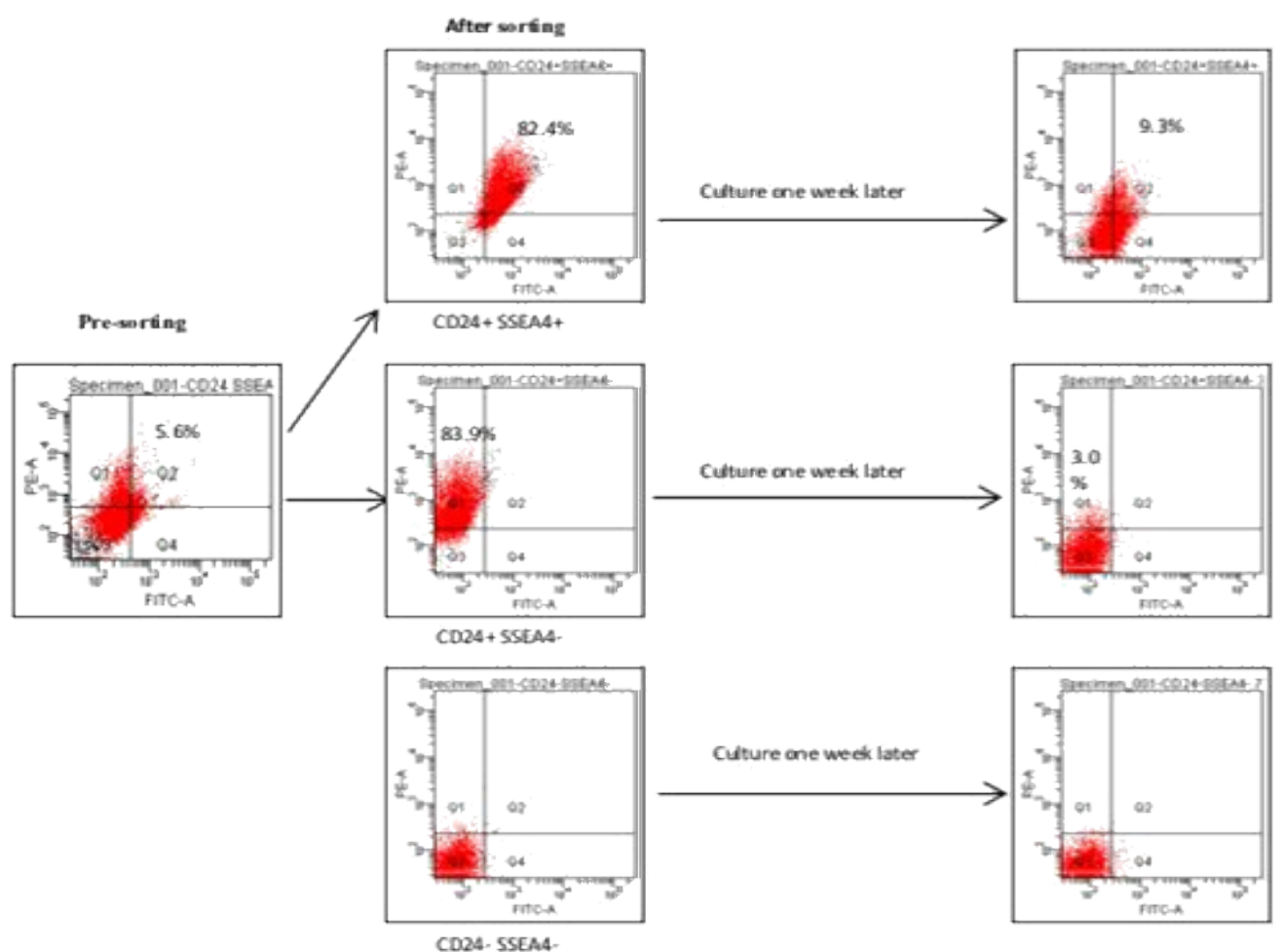

Figure 4: Self renewal activity of three sub-populations (CD24+SSEA4+, CD24+SSEA4-, CD24-) and presorted cells (parent cells). A: Differentiation of purified CD24+SSEA4+, CD24+SSEA4- and CD24-cells in subculture. Dot plots represent typical examples of CD24 and SSEA4 expression. The purity of the sorted population was $>96 \%$ as revealed by post-resorting analysis. After one week in culture, the purified CD24+SSEA4+ cells generated both CD24+SSEA4+, CD24+SSEA4- and CD24-cells, whereas others remained unchanged.

CD133-positive cells to be $21.1 \% \pm 5.6 \%$. However, when comparing the expression levels of stemness genes of CD133+ and CD133-cells, very little difference was observed. In contrast, SSEA4+ cells comprised $33.0 \% \pm 12.7 \%$, and comparison between the SSEA4+ and SSEA4-cells revealed that the genes oct 4, bmi-1, abcg-2, klf-4, and nanog were highly expressed in SSEA4+cells. CD24+ cells strongly expressed stemness genes, but CD24-cells did not express any of the genes, except for nestin. Thus, we concluded that CD24 and SSEA4 were markers for CSCs of OC.

It is known that CD24+SSEA4+cells are typical of CSCs due to the ability of CSCs to form spheres and to form colonies without any scaffold. Surprisingly, only 5 CD24+SSEA4+cells formed spheres in 3 weeks.

Furthermore, we demonstrated that the expansion of CD24+SSEA4+cells in a monolayer leads to the reconstitution of different sub-populations, similar to that found in the parent cells, whereas CD24+SSEA4- and CD24-SSEA4-cells were unable to repopulate the heterogeneity of the parent cells (Figure 4).

The expression of stem cell-related genes suggested that CD24+SSEA4+cells were more similar to stem cells than CD24+SSEA4cells. In addition, the CD24+SSEA4+cells might overexpress the DNMT3L gene, an enzymatically-inactive DNA methyltransferase. This is according to Gokul et al. who reported that increased expression of DNMT3L in primary OC stimulated conditions similar to partial iPS reprogramming [33]. Indeed, the most convincing proof of the identity of CSCs is their enhanced tumor-initiating capacity in vivo, which is universally considered the gold standard for evaluating CSCs.

The fact that we successfully serially transplanted human OCs in vivo in our experiments supported the hypothesis that these tumors comprised a sub-population of cells that were capable of self-renewal. When $1.0 \times 10^{2} \mathrm{CD} 24+$ SSEA4+cells/mouse were injected, all of the scid mice (4/4) were found to be tumorigenic within 2 months. In contrast, no tumors were observed after injection of $5 \times 10^{5} \mathrm{CD} 24$-cells. In fact, CD24+SSEA4+cells were 5000 times more tumorigenic than CD24cells.

Our results supported the hypothesis that cancer stem cells are resistant to chemotherapy and radiotherapy as these standard therapies fail to completely eradicate CSCs, resulting in recurrence. We showed that the CD24+SSEA4+cells were more resistant to cisplatin than the other types. In addition, CD24+SSEA4+cells were consistently the most tumorigenic of all the primary ovarian carcinoma cells. These results suggested that the CD24+SSE Despite many studies, markers of effective CSCs of heterogeneous ovarian carcinoma (OC) that can be directly used for medical treatment have not yet been reported. A4+cells in OC could survive exposure to existing anti-cancer therapies and cause recurrence. Thus, novel anti-cancer drugs targeting CSCs 
Citation: Wang F, Yoshida T, Okabe M, Fathy M, Sun Y, et al. (2017) CD24+SSEA4+cells in Ovarian Carcinoma Cells Demonstrated the Characteristics as Cancer Stem Cells. J Cancer Sci Ther 9: 343-352. doi: 10.4172/1948-5956.1000440

(a) Sphere formation assay

(a) -1 Morphology of sphere
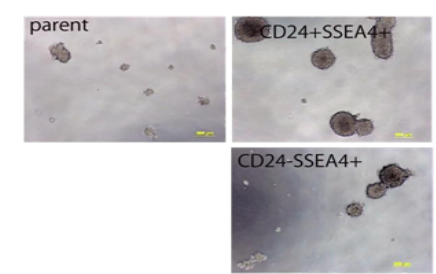

(a)-3 The distribution of size in differernat sub group.

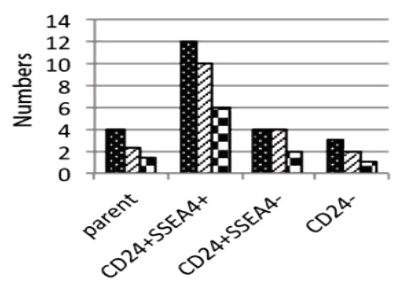

. $20-50 \mu \mathrm{m}$

口 $50-100 \mu \mathrm{m}$

घ $\mathrm{L}>100 \mu \mathrm{m}$ (a)-2 Numbers of Sphere

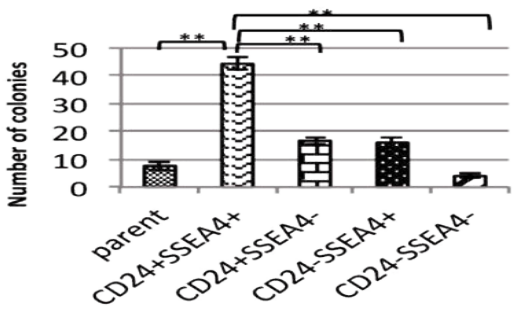

(a)-4 Sphere formation of small cell numbers

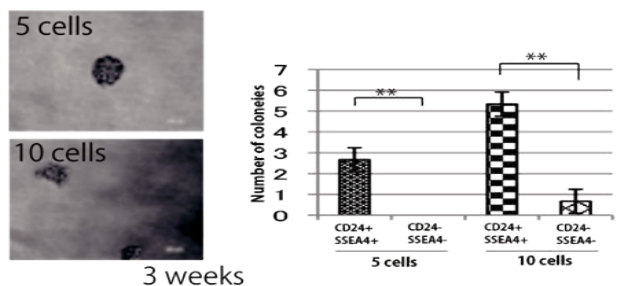

(b) Soft agar assay
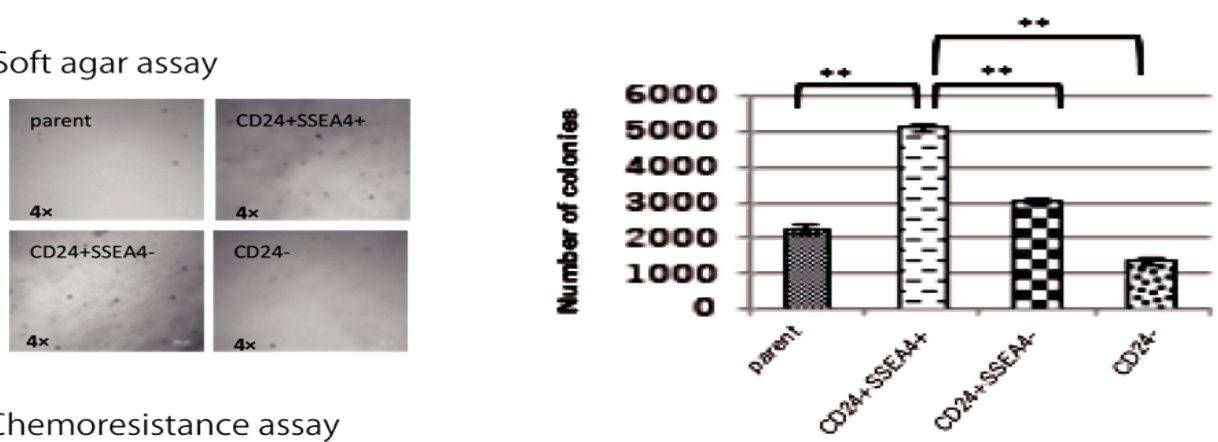

(c) Chemoresistance assay

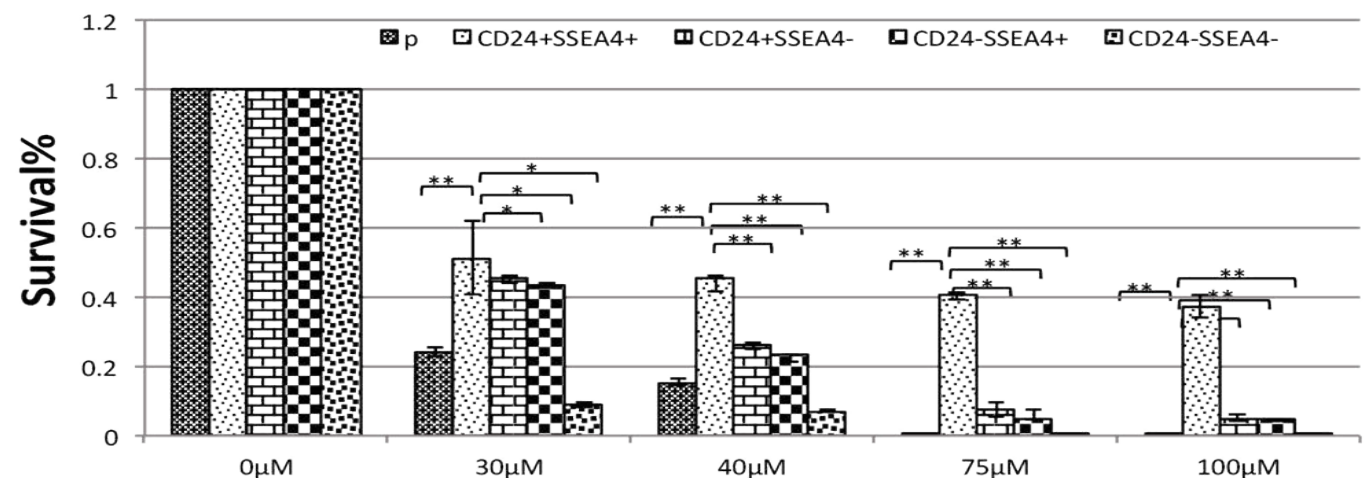

Figure 5: The characteristics of three sub-populations. The sorted sub-populations were tested for sphere formation using 24-well ultra-low attachment dishes and serum-free media.

(a)-1: Morphology of the sphere.

(a)-2: The CD24+SSEA4+ cells formed $44 \pm 2$ spheres per $1 \times 10^{3}$ cells and their average size was $200 \mu$ m. CD24-SSEA4-cells form small spheres under the same experimental conditions. The average diameter of the spheres was $20 \mu \mathrm{m}\left({ }^{* *} \mathrm{p}<0.01\right)$.

(a)-3: The formed spheres were divided into three type, large, middle and small $(L>100 \mu \mathrm{m}>\mathrm{M}>50 \mu \mathrm{m}>\mathrm{S}>20 \mu \mathrm{m})$. The spheres in the CD24+SSEA4+cells were most and biggest size in all sub-population.

(a)-4: The ability of sphere formation with small cell numbers. Only 5 or 10 cells of CD24+SSEA4+cells were made spheres within three weeks $\left({ }^{* *} p<0.01\right)$.

(b): The sorted cells were grown in soft agar. The CD24+SSEA4+ cells formed most

colonies in all sub-populations significantly $\left({ }^{* *} \mathrm{p}<0.01\right)$.

(c): Chemoresistance assay. The CD24+SSEA4+cells had kept the viability even if the concentration of cisplatin changed, it is viability was higher than other cells $\left({ }^{* *} p<0.01,{ }^{*} p<0.05\right)$. 
Citation: Wang F, Yoshida T, Okabe M, Fathy M, Sun Y, et al. (2017) CD24+SSEA4+cells in Ovarian Carcinoma Cells Demonstrated the Characteristics as Cancer Stem Cells. J Cancer Sci Ther 9: 343-352. doi: 10.4172/1948-5956.1000440
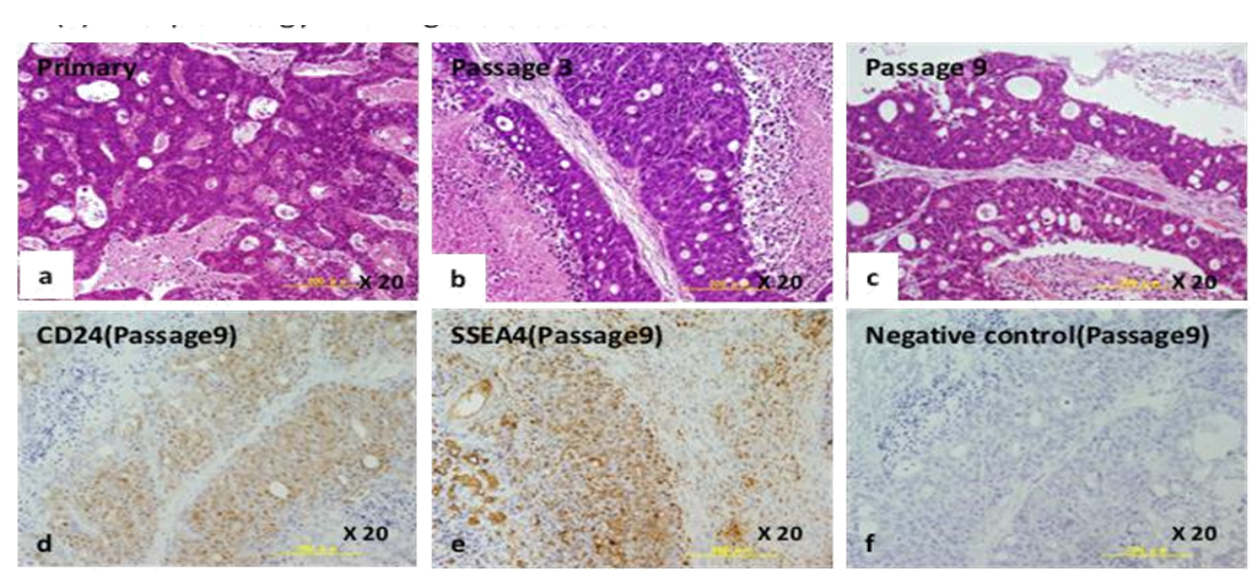

(b) Tumorgenicity

(b)-1 Incidence rate

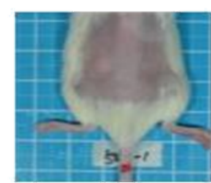

500 cells

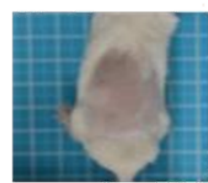

100 cells

\begin{tabular}{|l|c|c|c|c|}
\hline $\begin{array}{c}\text { Number of } \\
\text { Cells }\end{array}$ & $\begin{array}{c}\text { CD24+ } \\
\text { SSEA4+ }\end{array}$ & $\begin{array}{c}\text { CD24 } \\
\text { SSEA4+ }\end{array}$ & $\begin{array}{c}\text { CD24+ } \\
\text { SSEA4. }\end{array}$ & CD24- \\
\hline $5.0 \times 10^{5}$ & $3 / 3$ & - & - & $0 / 3$ \\
\hline $5.0 \times 10^{4}$ & $3 / 3$ & $0 / 3$ & - & $0 / 3$ \\
\hline $1.0 \times 10^{3}$ & $3 / 3$ & - & - & $0 / 3$ \\
\hline $5.0 \times 10^{2}$ & $5 / 5(8 / 8)$ & - & $1 / 8$ & $0 / 3$ \\
\hline $1.0 \times 10^{2}$ & $4 / 4$ & - & - & $0 / 3$ \\
\hline
\end{tabular}

(b)-2 Tumor formed from transplanted CD24+SSEA4+ cells
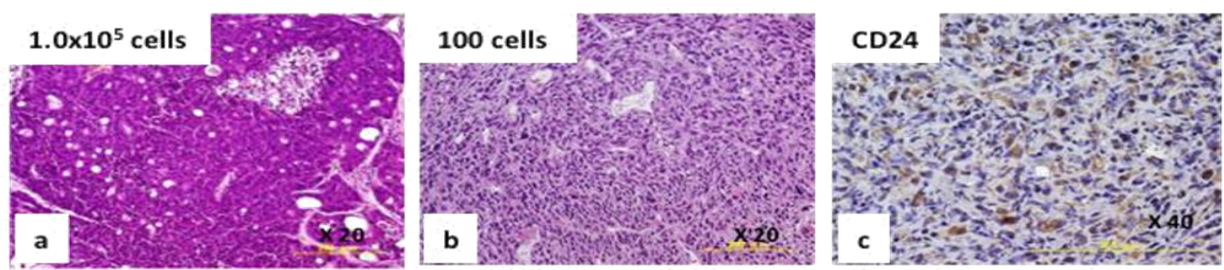

Figure 6: Histopathology of xenografted tumors. (a): Histopathology of xenografted tumor

Xenografted tumors in nude mice after passage 9 (c) presented same morphology as primary tumors, serous ovarian carcinoma (a). Immunohistochemistry, the cryo sections of the tumors from passage9 were stained with CD24 and SSEA4 (d,e). b: passage3, f: negative control without primary antibody. (b): Tumorigenicity of CD24+SSEA4+ cells in scid mice.

(b)-1: CD24+SSEA4+ or CD24- cells were transplanted subcutaneously into scid mice.

After three months it was found the tumor in the CD24+SSEA4+ cells transplanted mice. But no tumor observed in CD24- cells

(b)-2: Tumor formed from transplanted CD24+SSEA4+cells. The morphology of the tumor originated from $1.0 \times 10^{5}$ cells CD24+SSEA4+ cells was same morphology as primary tumor (a), but the tumor from 100 cells of CD24+SSEA4+cells were no regularity (b). Immunohistochemistry, it was concluded much CD24 positive cells (c).

need to be developed.

\section{Acknowledgements}

We would like to thank Ms. Furuichi for providing expert technical assistance.

\section{References}

1. Reya T, Morrioson SJ, Clarke MF, Weissman IL (2001) Stem cells, cancer, and cancer stem cells. Nature 414: 105-111.

2. Torino V, Desiderio V, Paino F, De Rosa A, Papaccio F, et al. (2013) Cancer stem cells in solid tumors: An overview and new approaches for their isolation and characterization. FASEB J 27: 13-24.

3. Cabanillas R, Llorente JL (2009) The stem cell network model: Clinical implications in cancer. Eur Arch Otorhinolaryngol 266(2): 161-170.

4. Eyler CE, Rich JN (2008) Survival of the fittest: Cancer stem cells in therapeutic resistance and angiogenesis. J Clin Oncol 10;26(17): 2839-2845.
5. Massard C, Deutsch E, Soria JC (2006) Tumour stem cell-targeted treatment Elimination or differentiation. Ann Oncol 17(11): 1620-1624.

6. Huang P, Wang CY, Gou SM, Wu HS, Liu T, et al. (2008) Isolation and biological analysis of tumor stem cells from pancreatic adenocarcinoma. World J Gastroenterol 14(24): 3903-3907.

7. Al-Hajj M, Wicha MS, Benito-Hernandez A, Morrison SJ, Clarke MF (2003) Prospective identification of tumorigenic breast cancer cells. Proc Natl Acad Sci USA 100(7): 3983-3988.

8. Ouhtit A, Abd Elmageed ZY, Abdraboh ME, Lioe TF, Raj MH (2007) In vivo evidence for the role of CD44s in promoting breast cancer metastasis to the liver. Am J Pathol 171(6): 2033-2039.

9. Bapat SA, Mali AM, Koppikar CB, Kurrey NK (2005) Stem and progenitor-like cells contribute to the aggressive behavior of human epithelial ovarian cancer Cancer Res 65(8): 3025-3029.

10. Szotek PP, Pieretti-Vanmarcke R, Masiakos PT, Dinulescu DM, Connolly D, 
Citation: Wang F, Yoshida T, Okabe M, Fathy M, Sun Y, et al. (2017) CD24+SSEA4+cells in Ovarian Carcinoma Cells Demonstrated the Characteristics as Cancer Stem Cells. J Cancer Sci Ther 9: 343-352. doi: 10.4172/1948-5956.1000440

et al. (2006) Ovarian cancer side population defines cells with stem cell-like characteristics and mullerian inhibiting substance responsiveness. Proc Nat Acad Sci U S A 103(30): 11154-11159.

11. Beba T, Convery PA, Matsumura N, Whitaker RS, Kondoh E, et al. (2009) Epigenetic regulation of CD133 and tumorigenicity of CD133+ ovarian cancer cells. Oncogene 28(2): 209-218.

12. Zhang S, Balch C, Chan MW, Lai HC, Matei D, et al. (2008) Identification and characterization of ovarian cancer-initiating cells from primary human tumors. Cancer Res 68(11): 4311-4320.

13. Alvelo AB, Chen R, Fu HH Montagna M, Schwartz PE, et al. (2009) Molecular phenotyping of human ovarian cancer stem cells unravels the mechanisms for repair and chemoresistance. Cell Cycle 8(1): 158-166.

14. Ince TA, Richardson AL, Bell GW Saitoh M, Godar S, et al. (2007) Transformation of different human breast epithelial cell types leads to distinct tumor phenotypes. Cancer Cell 12(2): 160-170.

15. Kurman RJ, Shih leM (2010) The origin and pathogenesis of epithelial ovarian cancer: A proposed unifying theory. Am J Surg Pathol 34(3): 433-443.

16. Dalerba P, Cho RW, Clarke MF (2007) Cancer stem cells: Models and concepts. Annu Rev Med 58: 267-284.

17. Clarke MF, Dick JE, Dirks PB, Eaves CJ, Jamieson CH, et al. (2006) Cancer stem cells-perspectives on current status and future directions: AACR workshop on cancer stem cells. Cancer Res 66: 9339-9344.

18. Hill RP, Perris R (2007) “Destemming” cancer stem cells. J Natl Cancer Inst 99: $1435-1440$

19. Hill RP (2006) Identifying cancer stem cells in solid tumors: Case not proven Cancer Res 66: 1891-1895.

20. Kelly PN, Dakic A, Adams JM, Nutt SL, Strasser A (2007) Tumor growth need not be driven by rare cancer stem cells. Science 317(5836): 337.

21. Yang XR, Xu Y, Yu B, Zhou U, Li JC, et al. (2009) CD24 is a novel predictor for poor prognosis of hepatocellular carcinoma after surgery. Clin Cancer Res 15(17): 5518-5527.
22. Nagy B, Szendroi A, Romics I (2009) Overexpression of CD24, c-myc and phospholipase $2 \mathrm{~A}$ in prostate cancer tissue samples obtained by needle biopsy. Pathol Oncol Res 15(2): 279-283.

23. Lee HJ, Kim DI, Kwak C, Ku JH, Moon KC (2008) Expression of CD24 in clea cell renal cell carcinoma and its prognostic significance. Urology 72(3): 603-677.

24. Gao MQ, Choi YP, Kang S, Youn JH, Cho NH (2010) CD24+ cells from hierarchically organized ovarian cancer are enriched in cancer stem cells. Oncogene 29(18): 2672-2680.

25. O'Brien CA, Pollett A, Gallinger S, Dick JE (2007) A human colon cancer cell capable of initiating tumour growth in immunodeficient mice. Nature 445(7123): 106-110.

26. Yin S, Li J, Hu C (2007) CD133 positive hepatocellular carcinoma cells possess high capacity for tumorigenicity. Int J Cancer 120(7): 1444-1450.

27. Rappa G, Fodstad O, Lorico A (2008) The stem cell-associated antigen CD133 (Prominin-1) is a molecular therapeutic target for metastatic melanoma. Stem Cells 26(2): 3008-3017.

28. Angelastro JM, Lame MW (2010) Overexpression of CD133 promotes drug resistance in C6 glioma cells. Mol Cancer Res 8(8): 1105-1115.

29. Ye F, Li Y, Hu Y, Zhou C, Hu Y, et al. (2010) Stage-specific embryonic antigen 4 expression in epithelial ovarian carcinoma. Int J Gynecol Cance 20(6): 958-964.

30. Noto Z, Yoshida T, Okabe M, Koike C, Fathy M, et al. (2013) CD44 and SSEA-4 positive cells in an oral cancer cell line HSC-4 possess cancer stem-like cell characteristics. Oral Oncol 49(8): 787-795.

31. Jaggupilli A, Elkord E (2012) Significance of CD44 and CD24 as cancer stem cell markers: an enduring ambiguity. Clin Dev Immunol 2012: Article ID 708036: 11.

32. Curley MD, Therrien VA, Cummings CL, Sergent PA, Koulouris CR, et al (2009) CD133 expression defines a tumor initiating cell population in primary human ovarian cancer. Stem Cells 27(12): 2875-2883.

33. Gokul G, Ramakrishna G, Khosla S (2009) Reprogramming of HeLa cells upon DNMT3L overexpression mimics carcinogenesis. Epigenetics 4(5): 322-329. 